\title{
Enumeration of Basic Hamilton Cycles in the Mangoldt Graph
}

\author{
Levaku Madhavi \\ Assistant Professor \\ Department of Applied Mathematics \\ Yogi Vemana University-Kadapa-516003 \\ Andhra Pradesh, India
}

\author{
Tekuri Chalapati \\ Department of Mathematics \\ Sree Vidyanikethan Engineering \\ College, Tirupati - 517502 \\ Andhra Pradesh, India
}

\begin{abstract}
The Mangoldt graph $M_{n}$ is an arithmetic function, namely, Mangoldt function $\wedge(n), n \geq 1$ an integer. In this paper the notion of a basic Hamilton cycles in $\mathrm{M}_{\mathrm{n}}$ is introduced and their number is enumerated.
\end{abstract}

\section{Keywords}

Mangoldt Graph, Hamilton Cycle, Basic Hamilton Cycles.

AMS(MOS) Subject Classification: 68R05

\section{Index Terms}

Graph Theory, Discrete Mathematics

\section{INTRODUCTION}

Nathanson [11] introduced the concepts of Number Theory, particularly, the theory of congruences into Graph Theory, which paved the way for the emergence of Arithmetic Graphs. Maheswari and Madhavi[8,9,10] introduced the Mangoldt graph $M_{n}$ which is an arithmetic graph associated with the Mangoldt function $\wedge(n), n \geq 1$, an integer. It is shown that $M_{n}$ is connected and neither bipartite nor a tree for $n \geq 6$. Further they have studied the vertex domination and edge domination and gave a formula for the number of triangles In this study the Hamiltonian nature of $\mathrm{M}_{\mathrm{n}}$ is established for various forms of $n$ by exhibiting the Hamilton cycles in the general setup and using these Hamilton cycles the notion of basic Hamilton cycle is introduced in $M_{n}$ and their number is enumerated.

\section{HAMILTONIAN PROPERTY OF THE MANGOLDT GRAPH MN}

Definition 2.1: Let $n \geq 1$ be an integer. The Mangoldt function $\wedge(n)$, is defined as follows:

$$
\Lambda(n)=\left\{\begin{array}{cc}
\log p, \text { if } n \text { is a power of prime } \\
0, & \text { otherwise }
\end{array}\right.
$$

For example, $\wedge(4)=\wedge\left(2^{2}\right)=\log 2 . \wedge(6)=0$

and $\wedge(8)=\wedge\left(2^{2}\right)=\log 2$.

Definition 2.2[1]: Let $n \geq 1$ be an integer. The Mangoldt graph $M_{n}$ is the graph whose vertex set is $\{1,2, \ldots, n\}$ and the edge set is $\{(\mathrm{x}, \mathrm{y}): \wedge(\mathrm{x} \cdot \mathrm{y})=0,1 \leq \mathrm{x}, \mathrm{y} \leq \mathrm{n}$ and $\mathrm{x} \neq \mathrm{y}$.

That is, the vertices $x$ and $y$ of are adjacent if and only if $\mathrm{x} \cdot \mathrm{y}$ is not a power of prime. Clearly $M_{n}$ is a simple undirected graph without loops.
Example 2.3: The graphs $M_{6}$ and $M_{7}$ are given below:

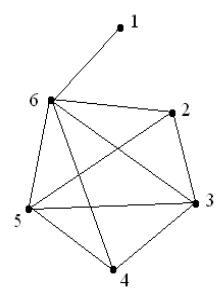

$\mathrm{M}_{6}$

Fig 2.2.1

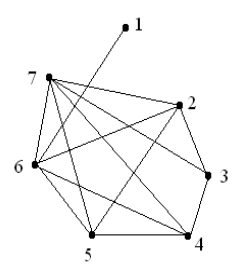

$\mathrm{M}_{7}$

Fig 2.2.2
Lemma 2.4: Let $n \geq 1$ be an integer. For all vertices $\mathrm{u}, 1<\mathrm{u}<\mathrm{n},(\mathrm{u}, \mathrm{u}+1)$ is an edge of $\mathrm{M}_{\mathrm{n}}$.

Proof: For all vertices $M_{n}$ in, one of $u$ and $u+1$ is even and the other odd. So $u(u+1)$ is not a power of a single prime so that there is an edge between $u$ and $u+1$.

It is evident that the vertex 1 is an isolated vertex in the graphs $\mathrm{M}_{1}, \mathrm{M}_{2}, \mathrm{M}_{3}, \mathrm{M}_{4}$ and $\mathrm{M}_{5}$ and thus these graphs are not Hamiltonian. In $\mathrm{M}_{6}, \mathrm{M}_{7}, \mathrm{M}_{8}$ and $\mathrm{M}_{9}$, the vertex 1 is adjacent only to the vertex 6 and hence its degree is one. So the vertex 1 does not belong to any cycle in these graphs, as the degree of a vertex in a cycle must be two. Thus these graphs do not contain Hamilton cycles and hence they are not Hamiltonian. So $M_{n}$ is not Hamiltonian for $n<10$. In the following it is established that for $\mathrm{n} \geq 10$ the graph $\mathrm{M}_{\mathrm{n}}$ is Hamiltonian. The following number theoretic result is needed.

Lemma 2.5 : For an integer $m>1,(m-1)(m+1)$ is not a power of a single prime.

Proof: If $m$ is odd then $m-1$ and $m+1$ are both even so that $\mathrm{m}-1=2 \mathrm{r}$ and $\mathrm{m}+1=2 \mathrm{r}+2=2(\mathrm{r}+1)$ for some positive integer $r$. So $(m-1)(m+1)=2^{2} r(r+1)$ and this is not a power of single prime since one of $r$ and $r+1$ is odd the other even.

If $m$ is even $m-1$ and $m+1$ are both odd. Suppose $(m-1)$ $(\mathrm{m}+1)=\mathrm{q}^{l}$ for some odd prime $\mathrm{q}$ and integer $l>1$. Then $\mathrm{m}-1=\mathrm{q}^{\mathrm{s}}$ and $\mathrm{m}+1=\mathrm{q}^{\mathrm{t}}$ for some integers $\mathrm{s}, \mathrm{t}, \mathrm{s}<\mathrm{t}, \mathrm{s} \geq 1$ and $\mathrm{s}+\mathrm{t}=l$. Also we have $\mathrm{q}^{\mathrm{t}}-\mathrm{q}^{\mathrm{s}}=2$ with $\mathrm{s}<\mathrm{t}$. Since $\mathrm{q}>2$, $\mathrm{q}^{\mathrm{t}}-\mathrm{q}^{\mathrm{s}}=2$ with $\mathrm{s}<\mathrm{t}$. Since $\mathrm{q}>2, \mathrm{q}^{\mathrm{t}}-\mathrm{q}^{\mathrm{s}}=\mathrm{q}^{\mathrm{s}}\left(\mathrm{q}^{\mathrm{t}-\mathrm{s}}-1\right)>2$, for all values of $\mathrm{q}, \mathrm{t}, \mathrm{s}$ which is a contradiction to $\mathrm{q}^{\mathrm{t}}-\mathrm{q}^{\mathrm{s}}=2$. So $(m-1)(m+1)$ is not a power of a single prime.

Theorem 2.6: For $n \geq 10$ and $n=2^{r}$, $r$ is a positive integer, $\mathrm{M}_{\mathrm{n}}$ is Hamiltonian.

Proof : Let $n \geq 10$ and $n=2^{r}, r$ a positive integer. There is no edge between 1 and 2 as well as 1 and $n$ in $M_{n}$ since $1 \times 2=2$ and $1 \times n=2^{r}$ are powers of the prime 2 . 
For positive integers 1 and $\mathrm{m}, l \neq \mathrm{m}$ which are less than $\mathrm{n}$ and not power of a single prime, consider the following vertex sequence in $M_{n}$.

$(2,3,4, \ldots, l-1, l+1, \ldots ., \mathrm{m}-1, \mathrm{~m}+1, \ldots ., \mathrm{n}-3, \mathrm{n}-2$, $\mathrm{n}, \mathrm{n}-1, l, 1, \mathrm{~m}, 2$ )

By the Lemma 2.4, there exist an edge between $u$ and $u+1$ for all vertices $\mathrm{u}, 1<\mathrm{u}<\mathrm{n}$, in $\mathrm{M}_{\mathrm{n}}$. By the Lemma 2.6, $(l-1)$ $(l+1)$ and $(\mathrm{m}-1)(\mathrm{m}+1)$ are not powers of a single prime. So there is an edge between $l-1$ and $l+1$ as well as $\mathrm{m}-1$ and $\mathrm{m}+1$. Also $\mathrm{n}(\mathrm{n}-2)=2^{\mathrm{r}}\left(2^{\mathrm{r}}-2\right)=2^{\mathrm{r}+1}\left(2^{\mathrm{r}-1}-1\right)$. This is a product of an even number $2^{\mathrm{r}+1}$ and an odd number $2^{\mathrm{r}+1}$ and an odd number $2^{\mathrm{r}-1}-1$ so that it is not a power of a single prime. So there is an edge between $n-2$ and $n$.

Since each of $l$ and $\mathrm{m}$ is not a power of a single prime. $(\mathrm{n}-1) \times l, l \times 1, l \times \mathrm{m}$ and $\mathrm{m} \times 2$ are also not powers of a single prime. Therefore $(\mathrm{n}-1, l),(l, 1),(l, \mathrm{~m})$ and $(\mathrm{m}, 2)$ are edges in $\mathrm{M}_{\mathrm{n}}$. Thus the vertex sequence ( $2,3,4, ., l-1$, $l+1, \ldots ., \mathrm{m}-1, \mathrm{~m}+1, \ldots ., \mathrm{n}-3, \mathrm{n}-2, \mathrm{n}, \mathrm{n}-1, l, 1, \mathrm{~m}, 2$ ) is a Hamilton cycle in $M_{n}$ and hence $M_{n}$ is Hamiltonian.

Example 2.7: For $\mathrm{n}=2^{4}=16$ the vertex sequences

(i) $(2,3,4,5,7,8,9,11,12,13,14,16,15,6,1,10,2)$

(ii) $(2,3,4,5,7,8,9,10,11,13,14,16,15,6,1,12,2)$

are Hamilton cycles in $\mathbf{M}_{16}$. The graphs of these Hamilton cycles are given below.



Hamilton cycle (i)

Fig. 2.2.3

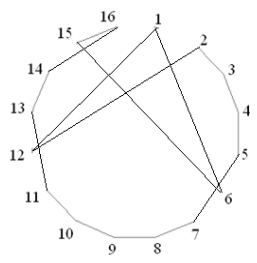

Hamilton cycle (ii)

Fig. 2.2.4
Theorem 2.8: For $n \geq 10$ and $n=p^{r}$, $p$ is a prime, $p \neq 2$ and $r$ a positive integer, the graph $M_{n}$ is Hamiltonian.

Proof: For $n \geq 10$ and $n=p^{r}, p$ is a prime, $p \neq 2$ and $r$ a positive integer. There is no edge between 1 and 2 since $1 \times 2$ is a power of 2 and there is no edge between 1 and $n$ since $1 \mathrm{x} \mathrm{n}=\mathrm{p}^{\mathrm{r}}$, $\mathrm{p}$ a prime.

Let $l$ and $\mathrm{m}, l \neq \mathrm{m}$ be a positive integers which are not powers of a single prime. Evidently $l \neq 1, \mathrm{n}$ and $\mathrm{m} \neq 1, \mathrm{n}$. Consider the following vertex sequence in $M_{n}$.

$(2,3,4, \ldots ., l-1, l+1, \ldots, \mathrm{m}-1, \mathrm{~m}+1, \ldots ., \mathrm{n}-2, \mathrm{n}-1$ $, \mathrm{n}, l, 1, \mathrm{~m}, 2)$.

By the Lemma 2.4 and 2.5, there edges in $\mathrm{M}_{\mathrm{n}}$ between the pairs $(l-1, l+1),(\mathrm{m}-1, \mathrm{~m}+1)$ and $(\mathrm{u}, \mathrm{u}+1), \mathrm{u} \in \mathrm{M}_{\mathrm{n}}$, $1<\mathrm{u}<\mathrm{n}$. Also there is an edge between $\mathrm{n}$ and 2 since $2 \times n=2 p^{r}, p \neq 2$.

Further $\mathrm{n} \times l, l \times 1, l \times \mathrm{m}$ and $\mathrm{m} \times 2$ are not powers of a single prime so that $(\mathrm{n}, l),(l, 1),(l, \mathrm{~m})$ and $(\mathrm{m}, 2)$ are edges in $\mathrm{M}_{\mathrm{n}}$. So the vertex sequence $(2,3,4, l-1, l+1$, $\ldots, \quad \mathrm{m}-1, \mathrm{~m}+1, \ldots ., \mathrm{n}-2, \mathrm{n}-1, \mathrm{n}, \quad l, 1, \mathrm{~m}, 2)$ is a Hamilton cycle in $M_{n}$ and $M_{n}$ is Hamiltonian.

Example 2.9: For $n=5^{2}=25$ the vertex sequences (i) $(2,3,4,5,7,8,9,10,11,12,13,14,15,16,17,18,19,21,22$, $23,24,25,6,1,20,2)$

(ii) $(2,3,4,5,7,8,9,10,11,12,13,14,15,16,17,18,19,20,21$, $22,23,25,6,1,24,2)$

are Hamilton cycles in $\mathrm{M}_{25}$.

Theorem 2.10 : For $\mathrm{n} \geq 10$ and $\mathrm{n}$ not a power of a single prime the graph $\mathrm{M}_{\mathrm{n}}$ is Hamiltonian.

Proof : Let $\mathrm{n} \geq 10$ and $\mathrm{n}$ not a power of a single prime. Let 1 and $\mathrm{m}, l \neq \mathrm{m}$ be positive integers less than or equal to $\mathrm{n}$, which are not powers of a single prime. The following three cases will arise.

Case (1) : Let $l \neq \mathrm{m}$ and $\mathrm{m} \neq \mathrm{n}$. As in the Theorem 2.8 one can see that the vertex sequence

$(2,3,4, \ldots ., l-1, l+1, \ldots ., \quad \mathrm{m}-1, \mathrm{~m}+1, \ldots ., \quad \mathrm{n}-2$, $\mathrm{n}-1, \mathrm{n}, \quad l, 1, \mathrm{~m}, 2)$ is a Hamilton cycle in $\mathrm{M}_{\mathrm{n}}$.

Case (2) : Let $l=\mathrm{n}$ and $\mathrm{m} \neq \mathrm{n}$. The following two sub cases will arise.

Subcase (1) : Let $n-1$ be not a power of 2. It is easy to see that the vertex sequence

$(2,3,4, \ldots, \mathrm{m}-1, \mathrm{~m}+1, \ldots, \mathrm{n}-2, \mathrm{n}-1, \mathrm{n}, 1, \mathrm{~m}, 2)$ is a Hamilton cycle in $M_{n}$.

Subcase (2) : Let $\mathrm{n}-1$ be a power of 2 . Consider the following vertex sequence in $\mathrm{M}_{\mathrm{n}}(2,3,4, \ldots, \mathrm{m}-1, \mathrm{~m}+1$, ,., $\mathrm{n}-3, \mathrm{n}-1, \mathrm{n}-2, \mathrm{n}, 1, \mathrm{~m}, 2)$.

Clearly the pairs $(2,3),(3,4), \ldots \ldots .(m-1, m+1) \ldots .$. $(\mathrm{n}-1, \mathrm{n}-2),(\mathrm{n}-2, \mathrm{n})(\mathrm{n}, 1),(l, \mathrm{~m})$ and $(\mathrm{m}, 2)$ are adjacent in $M_{n}$. The vertices $n-2$ and $n-1$ are also adjacent if $(n-3)(n-1)$ is not a power of a single prime. Suppose that $(n-3)(n-1)$ is not a power of a single prime. Suppose that $(n-3)(n-1)=p^{r}$ for some prime $p$ and $r$ a positive integer. Then $\mathrm{n}-3=\mathrm{p}^{\mathrm{s}}$ for some positive integers $\mathrm{s}$, $\mathrm{t}$ and $\mathrm{s}<\mathrm{t}$. This gives $\mathrm{p}^{\mathrm{t}}-\mathrm{p}^{\mathrm{s}}=(\mathrm{n}-1)-(\mathrm{n}-3)=2$ which is true only for the least values of $p, s$ and $t$ namely, $p=2$, $\mathrm{s}=1$ and $\mathrm{t}=2$. But for $\mathrm{p}=2, \mathrm{~s}=1$ and $\mathrm{t}=2$ we have $\mathrm{n}-3=$ $\mathrm{p}^{\mathrm{s}}=2$, or, $\mathrm{n}=5$ which is a contradiction to $\mathrm{n} \geq 10$. So $(n-3)(n-1)$ is not a power of a single prime and thus $n-2$ and $\mathrm{n}-1$ are adjacent in $\mathrm{M}_{\mathrm{n}}$. These show the vertex sequence.

$(2,3,4, ., \mathrm{m}-1, \mathrm{~m}+1, ., \mathrm{n}-3, \mathrm{n}-1, \mathrm{n}-2, \mathrm{n}, 1, \mathrm{~m}, 2)$ is a Hamilton cycle in $M_{n}$.

Case (3) : Let $l \neq \mathrm{n}$ and $\mathrm{m}=\mathrm{n}$.

Subcase (1): Let $n-1$ be not a power of 2. As in Subcase (1) of Case (2), it is easy to see that the vertex sequence

$(2,3,4, \ldots, l-1, l+1, ., \mathrm{n}-3, \mathrm{n}-3, \mathrm{n}-1 \quad l, 1, \mathrm{n}, 2)$ is a Hamilton cycle in $M_{n}$.

Subcase (2) : Let $n-1$ be a power of 2. Again as in Subcase (2) of Case (2), one can see that the vertex sequence

$(2,3,4, ., l-1, l+1, \ldots, \mathrm{n}-3, \mathrm{n}-1, \mathrm{n}-2, l, 1, \mathrm{n}, 2)$ is a Hamilton cycle in $M_{n}$. So $M_{n}$ is Hamiltonian in this case also.

Example 2.11 : For $n=15$ the vertex sequences 
(i) $(2,3,4,5,7,8,9,11,12,13,14,15,6,1,10,2)$

(ii) $(2,3,4,5,6,7,8,9,11,12,13,14,15,1,10,2)$

are Hamilton cycle in $\mathrm{M}_{15}$.

\section{ENUMERATION OF BASIC HAMILTON CYCLES IN Mn}

In this Section the concept of basic Hamilton cycle in the Mangoldt graph $M_{n}$ is introduced and the number of basic Hamilton cycles in $M_{n}$ is determined for various forms of $n$.

Definition 3.1: Let $n \geq 10$ be an integer. A Hamilton cycle of the form

$(. ., l-1, l+1, \ldots . ., \mathrm{m}-1, \mathrm{~m}+1, \ldots ., l, 1, \mathrm{~m}, \ldots .$.$) where l$ and $\mathrm{m}$ are positive integers less than are equal to $\mathrm{n}$, which are not powers of a sing prime, is called a basic Hamilton cycle in the Mangoldt graph $M_{n}$.

The Hamilton cycles given in the Theorem 2.6, 2.8 and 2.10 are examples of the basic Hamilton cycles in $M_{n}$ for various forms of $n$.

Lemma 3.3 : Let $\mathrm{n}$ be a positive integer. The number $\mathrm{N}$ of positive integers less than or equal to $n$ which are not powers of a single prime is equal to $n-\left(\alpha_{1}+\alpha_{2}+.+\alpha_{k}+1\right)$, where $\mathrm{p}_{1}<\mathrm{p}_{2}<\ldots<\mathrm{p}_{\mathrm{k}} \leq \mathrm{n}$ are primes and $\alpha_{1}, \alpha_{2}, \ldots, \alpha_{\mathrm{k}}$ are the largest positive integers such that $p_{i}^{\alpha} \mathrm{i} \leq \mathrm{n}, l \leq \mathrm{i} \leq \mathrm{k}$.

Proof: Let $\mathrm{n}$ be a positive integer and let $\mathrm{p}_{1}<\mathrm{p}_{2}<\ldots<\mathrm{p}_{\mathrm{k}}$ be primes and $\alpha_{1}, \alpha_{2}, \ldots, \alpha_{k}$ be the largest positive integers such that $p_{i}^{\alpha} i \leq \mathrm{n}, l \leq \mathrm{i} \leq \mathrm{k}$, the powers of the prime $p_{\mathrm{i}}$ which are less than or equal to $\mathrm{n}$ are $p_{i}^{1}, p_{i}^{2}, \ldots, p_{i}^{\alpha} i$, and their number is equal to $\alpha_{i}$. Deleting these $\alpha_{1}, \alpha_{2}, \ldots, \alpha_{k}$ number of positive integers, which are powers of a single prime from 1,2 , $3, \ldots \ldots, \mathrm{n}$ we get 1 and the positive integers less than or equal to $\mathrm{n}$ which are not powers of a single prime. So number of positive integers less than or equal to $\mathrm{n}$ which are not powers of a single prime equal to $\mathrm{n}-\left(\alpha_{1}, \alpha_{2}, \ldots, \alpha_{\mathrm{k}}+1\right)=\mathrm{N}$.

Theorem 3.4 : For $n \geq 10$ and $n=2^{r}$, $r$ a positive integer, the number of basic Hamilton cycles in the Mangoldt graph $\mathrm{M}_{\mathrm{n}}$ is equal to $(\mathrm{n}-3) \times{ }^{\mathrm{N}} P_{2}$.

Proof: Let $n \geq 10$ and $n=2^{r}, r$ a positive integer. By the Theorem 2.6, the cycle
$(2,3,4, \ldots ., l-1, l+1, \ldots ., \mathrm{m}-1, \mathrm{~m}+1$,
$\mathrm{n}-3, \mathrm{n}-2$,
$\mathrm{n}, \mathrm{n}-1, l, 1, \mathrm{~m}, 2) \ldots \ldots$

is a basic Hamilton cycle in $\mathrm{M}_{\mathrm{n}}$, where $l$ and $\mathrm{m}$, are positive integers less than $\mathrm{n}$ which are not powers of a single prime.

Clearly $l \neq \mathrm{n}$ and $\mathrm{m} \neq \mathrm{n}$. By the Lemma 5.4 .3 the number of positive integers $\leq \mathrm{n}$ which are not powers of a single prime is equal to $\mathrm{N}=\mathrm{n}-\left(\alpha_{1}+\alpha_{2}+\ldots .+\alpha_{\mathrm{k}}+1\right)$ where $\alpha_{\mathrm{i}}, 1 \leq \mathrm{i} \leq \mathrm{k}$, are positive integers such that $p_{i}^{\alpha} i \leq \mathrm{n}$ for primes $\mathrm{p}_{1}<\mathrm{p}_{2}<\ldots$, $\mathrm{p}_{\mathrm{k}} \leq \mathrm{n}$.

For every choice of $l$ and $\mathrm{m}$ in triad $(l, 1, \mathrm{~m})$ from this collection of $\mathrm{N}$ positive integers which are not powers of a single prime there is a basic Hamilton cycle of the form (3.1). There are ${ }^{\mathrm{N}} \mathrm{P}_{2}$ choices for $l$ and $\mathrm{m}$ from the above $\mathrm{N}$ positive integers. So the number of basic Hamilton cycles of the form (3.1) in the Mangoldt graph $\mathrm{M}_{\mathrm{n}}$ is equal to ${ }^{\mathrm{N}} P_{2}$.

Taking any one these ${ }^{\mathrm{N}} P_{2}$ basic Hamilton cycles and replacing the triad $(l, 1, \mathrm{~m})$ in any one of the $\mathrm{n}-3$ places between 2,$3 ; 3,4 ; \ldots ; l-1, l+1, \ldots ; \mathrm{m}-1, \mathrm{~m}+1 ; \ldots ; \mathrm{n}-2, \mathrm{n} ; \mathrm{n}$, $\mathrm{n}-1$ and $\mathrm{n}, 2$ in this basic Hamilton cycle one gets the following $\mathrm{n}-3$ basic Hamilton cycles, since $(\mathrm{n}-1) 2=$ $\left(2^{\mathrm{r}}-1\right) 2$ and this is a product of an odd number $2^{\mathrm{r}}-1$ and an even number 2 so that it is not a power of a single prime and there is an edge between $n-1$ and 2 .

$(2, l, 1, \mathrm{~m}, 3,4, \ldots, l-1, l+1, \ldots, \mathrm{m}-1, \mathrm{~m}+1, \ldots, \mathrm{n}-3$, $\mathrm{n}-2, \mathrm{n}, \mathrm{n}-1,2)$,

$(2,3, l, 1, \mathrm{~m}, 4, \ldots, l-1, l+1, \ldots, \mathrm{m}-1, \mathrm{~m}+1, \ldots, \mathrm{n}-3$, $\mathrm{n}-2, \mathrm{n}, \mathrm{n}-1,2)$,

$(2,3,4, \ldots, l-1, l, 1, \mathrm{~m}, l+1, \ldots, \mathrm{m}-1, \mathrm{~m}+1, \ldots, \mathrm{n}-3$, $\mathrm{n}-2, \mathrm{n}, \mathrm{n}-1,2)$,

$(2,3,4, \ldots l-1, l+1, \ldots, \mathrm{m}-1, l, 1, \mathrm{~m}, \mathrm{~m}+1, \ldots, \mathrm{n}-3$, $\mathrm{n}-2, \mathrm{n}, \mathrm{n}-1,2$ ),

$(2,3,4, \ldots, l-1, l+1, \ldots, \mathrm{m}-1, \mathrm{~m}+1, \ldots, \mathrm{n}-3, \mathrm{n}-2$, $\mathrm{n}, \mathrm{n}-1, l, 1, \mathrm{~m}, 2$ )

So that the number of basic Hamilton cycles is in $M_{n}$ equal to $(\mathrm{n}-3) \times{ }^{\mathrm{N}} P_{2}$

Example 3.5 : Consider the Mangoldt graph $\mathrm{M}_{16}$. Here $\mathrm{n}=16=2^{4}$ and $\mathrm{n}-1=15$ which is not a prime power. Also $\alpha_{1}=4, \alpha_{2}=2, \alpha_{3}=\alpha_{4}=\alpha_{5}=\alpha_{6}=1$. So the number of positive integers less than 16 which are not powers of a single prime and 1 is equal to $\mathrm{N}=16-(4+2+1+1+1+1+1)=$ $16-11=5$ and the number of basic Hamilton cycles in the Mangoldt graph $\mathrm{M}_{16}$ is equal to

$(\mathrm{n}-3) \times{ }^{\mathrm{N}} P_{2}=(16-3) \times{ }^{5} P_{2}=13 \times 5 \times 4=260$.

Theorem 3.6 : Let $n \geq 10$ be an integer such that $n=p^{r}$, $p$ a prime $p \neq 2$ and $r$ a pisitive integer. The number of basic Hamilton cycles in the Mangoldt graph $M_{n}$ is equal to $(\mathrm{n}-3) \mathrm{x}^{\mathrm{N}} P_{2}$.

Proof: Let $n \geq 10$ be an integer such that $n=p^{r} p$ a prime , $\mathrm{p} \neq 2$ and $\mathrm{r}$ positive integer. By the Theorem 2.8 , the cycle

$(2,3,4, \ldots, l-1, l+1, \ldots, \mathrm{m}-1, \mathrm{~m}+1, \quad \mathrm{n}-2, \mathrm{n}-1$ $\mathrm{n}, l, 1, \mathrm{~m}, 2) \ldots \ldots$.

is a basic Hamilton cycle in $\mathrm{M}_{\mathrm{n}}$, where $l$ and $\mathrm{m}$ are positive integers less than or equal to $\mathrm{n}$ which are not primes of a single prime. Clearly $l \neq \mathrm{n}$ and $\mathrm{m} \neq \mathrm{n}$.

As in Theorem 3.4 one can see that the number of basic Hamilton cycles of the form 3.2, in the Mangoldt graph $M_{n}$ is equal to ${ }^{\mathrm{N}} P_{2}$ Choosing any one these ${ }^{\mathrm{N}} P_{2}$ basic Hamilton cycles and replacing the triad $(l, 1, \mathrm{~m})$ in any one of the $\mathrm{n}-3$ places between

2,$3 ; 3,4 ; \ldots ; l-1, l+1, \ldots ; \mathrm{m}-1, \mathrm{~m}+1 ; \ldots ; \mathrm{n}-2$, $\mathrm{n}-1 ; \mathrm{n}-1, \mathrm{n}$, and $\mathrm{n}, 2$ in this basic Hamilton cycle one gets the following $n-3$ basic Hamilton cycles, since $n \times 2=p^{r} \times 2$, $\mathrm{p} \neq 2$ and this not a power of a single prime so that there is an edge between $n$ and 2 .

$(2, l, 1, \mathrm{~m}, 3,4, \ldots, l-1, l+1, \ldots, \mathrm{m}-1, \quad \mathrm{~m}+1, \ldots$, $\mathrm{n}-2, \mathrm{n}-1, \mathrm{n}, 2)$,

$(2,3, l, 1, \mathrm{~m}, 4, \ldots, l-1, l+1, \ldots, \mathrm{m}-1, \quad \mathrm{~m}+1, \ldots$, $\mathrm{n}-2, \mathrm{n}-1, \mathrm{n}, 2)$, 
$(2,3,4, \ldots ., l-1, l, 1, \mathrm{~m}, l+1, \ldots, \mathrm{m}-1, \mathrm{~m}+1, \ldots, \mathrm{n}-2$, $\mathrm{n}-1, \mathrm{n}, 2)$,

$(2,3,4, \ldots l-1, l+1, \ldots, \mathrm{m}-1, l, 1, \mathrm{~m}, \mathrm{~m}+1, \ldots, \mathrm{n}-2, \mathrm{n}$ $-1, \mathrm{n}, 2)$,

$(2,3,4, \ldots, l-1, l+1, \ldots, \mathrm{m}-1, \mathrm{~m}+1, \ldots, \mathrm{n}-2, \mathrm{n}-1$, $\mathrm{n}, l, 1, \mathrm{~m}, 2$ ).

So the number of cycles is in $\mathrm{M}_{\mathrm{n}}$ equal to $(\mathrm{n}-3) \mathrm{x}{ }^{\mathrm{N}} P_{2}$.

Example 3.7 : Consider the Mangoldt graph $\mathrm{M}_{25}$. Here $\mathrm{n}$ $=25=5^{2}$. Also $\alpha_{1}=4, \alpha_{2}=2, \quad \alpha_{3}=2, \alpha_{4}=\alpha_{5}=\alpha_{6}=\alpha_{7}=$ $\alpha_{8}=\alpha_{9}=1$. The number $\mathrm{N}$ of positive integers less than 25 which are not a powers of a single prime and 1 is given by

$\mathrm{N}=25-(4+2+21+1+1+1+1+1+1)=25-15=10$ thus the number of basic Hamilton cycles in the Mangoldt graph $\mathrm{M}_{25}$ is equal to

$(\mathrm{n}-3) \times{ }^{\mathrm{N}} P_{2}=(25-3) \times{ }^{10} P_{2}=22 \times 10 \times 9=1980$.

Theorem 3.8 : Let $n \geq 10$ be an integer which is not a power of a single prime. The number of basic Hamilton cycles in the Mangoldt graph $\mathrm{M}_{\mathrm{n}}$ is equal to $(\mathrm{n}-3) \mathrm{x}{ }^{\mathrm{N}-1} P_{2}+$ $2(n-2)(N-1)$.

Proof: Let $n \geq 10$ be an integer which is not a power of a single prime.

Let $l \leq \mathrm{n}$ and $\mathrm{m} \leq \mathrm{n}, l \neq \mathrm{m}$ be positive integers which are not powers of a single prime.

Case (1): Let $l \leq \mathrm{n}$ and $\mathrm{m} \leq \mathrm{n}$.

In the Theorem 2.10, we have seen that $(2,3,4, \ldots, l-1, l+$ $1, \ldots, \mathrm{m}-1, \mathrm{~m}+1, \mathrm{n}-2, \mathrm{n}-1, \mathrm{n}, l, 1, \mathrm{~m}, 2) \ldots(3.3)$

is a basic Hamilton cycle in $M_{n}$,

By the Lemma 3.3, the number of positive integers $\leq \mathrm{n}$ which are not powers of a single prime and 1 is equal to $\mathrm{N}=\mathrm{n}-\left(\alpha_{1}+\alpha_{2}+\ldots .+\alpha_{\mathrm{k}}+1\right)$ where $\alpha_{\mathrm{i}}, 1 \leq \mathrm{i} \leq \mathrm{k}$, are positive integers $<\mathrm{n}($ since $l \neq \mathrm{n}$ and $\mathrm{m} \neq \mathrm{n})$ which are not powers of a single prime is equal to $\mathrm{N}-1$. The number of basic Hamilton cycle of the form (3.3) are got by choosing all possible $l$ and $\mathrm{m}$ in triad $(l, 1, \mathrm{~m})$ from these $\mathrm{N}-1$ positive integers $<\mathrm{n}$ which are not powers of a sing prime. Thus the number of basic Hamilton cycles of the form ( 3.3) in the Mangoldt graph $\mathrm{M}_{\mathrm{n}}$ is equal to ${ }^{(\mathrm{N}-1)} P_{2}$. Choosing any one of these ${ }^{(\mathrm{N}-1)} P_{2}$ basic Hamilton cycles and replacing the triad $(l, 1, \mathrm{~m})$ in any one of the $\mathrm{n}-3$ places between 2,$3 ; 3,4$; $\ldots ; l-1, l+1, \ldots ; \mathrm{m}-1, \mathrm{~m}+1 ; \ldots ; \mathrm{n}-2, \mathrm{n}-1 ; \mathrm{n}-1, \mathrm{n}$ and $n, 2$ we get $n-3$ basic Hamilton cycles. Thus the number of basic Hamilton cycles in $M_{n}$ is equal to $(n-3) x$ ${ }^{(\mathrm{N}-1)} P_{2}$.

Case (2): Let $l=\mathrm{n}$ and $\mathrm{m} \neq \mathrm{n}$.

Subcase (1) : Let $\mathrm{n}-1$ be not a power of 2 .

By the Subcase (1) of Case (2) of the Theorem 2.10, the cycle

$(2,3,4, . ., \mathrm{m}-1, \mathrm{~m}+1, \ldots, \mathrm{n}-2, \mathrm{n}-1, \mathrm{n}, \mathrm{l}, \mathrm{m}, 2) \ldots(3.4)$

is a basic Hamilton cycle in $M_{n}$, where $m \neq n$ is a positive integer which is not a power of single prime.
Since the number of positive integers $\mathrm{m}<\mathrm{n}$ which are not powers of a single prime is equal to $\mathrm{N}-1$, the total number of basic Hamilton cycle of the form (3.4) in Mangoldt graph $\mathrm{M}_{\mathrm{n}}$ is equal to $\mathrm{N}-1$. It is easy to see that ( since $\mathrm{n}-1$ is not a power of 2 ) by replacing the triad $(\mathrm{n}, l, \mathrm{~m})$ in any one of the $\mathrm{n}-2$ places between 2,$3 ; 3,4 ; \ldots ; \mathrm{m}-1, \mathrm{~m}+1 ; \ldots$; $\mathrm{n}-2, \mathrm{n}-1$; and $\mathrm{n}-1,2$ of any one the $\mathrm{N}-1$ basic Hamilton cycles of the form ( 3.4 ) one gets the following $n-$ 2 basic Hamilton cycles so that the number of basic Hamilton cycles in $M_{n}$ is equal to $(n-2) \quad(N-1)$.

Subcase (2) : Let $\mathrm{n}-1$ be a power of 2 .

By the Subcase (2) of Case (2) of the Theorem 2.10, the cycle

$(2,3,4, ., \mathrm{m}-1, \mathrm{~m}+1, ., \mathrm{n}-3, \mathrm{n}-1, \mathrm{n}-2, \mathrm{n}, l, \mathrm{~m}, 2) \ldots(3.5)$ is a basic Hamilton cycle in $M_{n}$, where $m \neq n$ is a not a power of a single prime and $n-1$ is a power of 2. As in Subcase (1) the number of basic Hamilton cycles of the form ( 3.5) in $M_{n}$ is equal to $\mathrm{N}-1$. By replacing the triad $(\mathrm{n}, l, \mathrm{~m})$ in any one of the $\mathrm{n}-2$ places between 2,$3 ; 3,4 ; \ldots ; \mathrm{m}-1, \mathrm{~m}+1 ; \ldots$; $\mathrm{n}-3, \mathrm{n}-1 ; \mathrm{n}-1, \mathrm{n}-2$ and $\mathrm{n}-2,2$ of in any of the $\mathrm{N}-1$ basic Hamilton cycles of the form ( 3.5 ) one gets the following $\mathrm{n}-2$ basic Hamilton cycles, since $\mathrm{n}-1$ is power of $2,(\mathrm{n}-2) 2=\left(2^{\mathrm{s}}-1\right) 2$ for some integer $\mathrm{s}>1$ and this is not a power of a single prime since $2^{\mathrm{s}}-1$ is odd and 2 is even so that $(n-2,2)$ is an edge in $M_{n}$. The number of basic Hamilton cycles in $M_{n}$ is equal to $(n-2)(N-1)$.

Case (3): Let $l \neq \mathrm{n}$ and $\mathrm{m} \neq \mathrm{n}$.

As in the Case (2) one can see that the number of basic Hamilton cycles in $M_{n}$ is equal to $(n-2)(N-1)$.

From these three cases it follows that when $\mathrm{n}$ is not a power of a single prime the number of basic Hamilton cycles in the Mangoldt graph $M_{n}$ is equal to

$(\mathrm{n}-3) \mathrm{x}^{(\mathrm{N}-1)} \mathrm{P}_{2}+(\mathrm{n}-2)(\mathrm{N}-1)+(\mathrm{n}-2)(\mathrm{N}-1)$

$=(\mathrm{n}-3) \mathrm{x}^{(\mathrm{N}-1)} P_{2}+2(\mathrm{n}-2)(\mathrm{N}-1)$.

Example 3.9 : Consider the Mangoldt graph $\mathrm{M}_{15}$. Here $\mathrm{n}=15$ Also $\alpha_{1}=3, \alpha_{2}=2, \alpha_{3}, \alpha_{4}=\alpha_{5}=\alpha_{6}=1$. The number of positive integers less than or equal to 50 which are not powers of a single prime and 1 is given by

$\mathrm{N}=15-(3+2+1+1+1+1+1)=15-10=5$ and the number of basic Hamilton cycles in the Mangoldt graph $\mathrm{M}_{15}$ is equal to $(\mathrm{n}-3) \mathrm{x}^{(\mathrm{N}-1)} P_{2}+2(\mathrm{n}-2)(\mathrm{N}-1)=(15-3) \mathrm{x}$ ${ }^{(5-1)} P_{2}+2(15-2)(5-1)=12 \times 4 \times 3+2 \times 13 \times 4=144+$ $104=248$.

Conclusion : The basic Hamilton Cycles Enumerated in section 3 are not disjoint and their number is too large. It will be interesting to find out the number of disjoint basic Hamilton Cycles in the Mangoldt Graph $\mathrm{M}_{\mathrm{n}}$ for given integer $\mathrm{n} \geq 1$, in which case their number will be less and easy to handle.

\section{ACKNOWLEDGEMENT}

The authors express their thanks to Prof. L. Nagamuni Reddy for his suggestions during the preparation of this paper and the referee for his suggestions.

\section{REFERENCES}

[1] Apostol, T. M., - Introduction to Analytic Number Theory, Springer international student Edition (1989). 
[2] Berrizbeitia P. and Giudici R.E., - Counting pure k cycles in sequences of Cayley Graphs, Discrete Math., 149, 11-18 (1996).

[3] Berrizbeitia P. and Giudici R.E.,; - On Cycles in the sequence of unitary Cayley graphs. Reporte Techico No.01-95, Universibad Simon Bolivar, Dpto, de Mathematics, Caracas, Venezuela (1995).

[4] Bondy J.A. and Murty U.S.R.; - Graph Theory with Applications, Macmillan, London (1976).

[5] Dejter I. and Giudici R.E. ; On unitary Cayley graphs, JCMCC, 18 121-124 (1995).

[6] Dickson E. - History of Theory of Numbers, Vol.1, Chelsea Publishing Company (1952).

[7] Madhavi L. and Maheswari B.; - Enumeration of Hamilton cycles and triangles in quadratic residue Cayley graphs, Chamchuri J.Math., 1(1), 95-103 (2009).
[8] Madhavi, L., and Maheswari, B., - Edge cover Domination in Mangoldt Graph, MEJS, 3(1): 37-51, (2011).

[9] Maheswari, B., and Madhavi, L., - Vertex Domination in Mangoldt Graph, Journal of APSMS, Vol(1), NO.2, pp 184-190, (2008).

[10] Maheswari, B., and Madhavi, L., - Counting of Triangles in Mangoldt Graph, J.Pure and Appl., Phys. Vol.20, NO.3, pp. 165-169, July-Sep (2008).

[11] Nathanson, B. Melvyn., - Connected Components of Arithmetic Graphs, Monat. fur.Math, 29(1980).

[12] Vasumathi, N., - Number Theoretic Graphs, Doctoral Thesis submitted to S.V.University, Tirupati, India, (1994). 